\title{
35. Studies on the Regeneration of Earthworms by Transplantation.
}

\section{Influence of the Nervous System.}

\author{
By Yô K. OKADA ${ }^{1)}$ and Itue KaWAKami. \\ Zoological Institute, Kyoto Imperial University. \\ (Comm. by N. YATSU, M.I.A., May 12, 1941.)
}

In the earthworm Eisenia foetida, new formation of segments can be induced at any level either by deflection of the ventral nerve cord or by implantation of a piece of it under the body wall. In Fig. 1 is shown the formation of new segments on the dorsal surface of the body obtained with the method of deflection, and Fig. 2 represents that

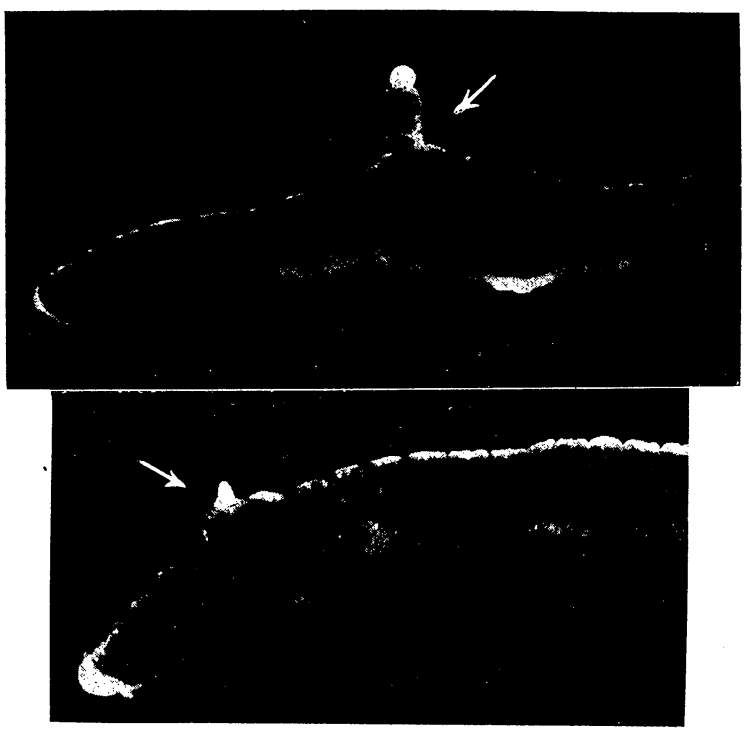

Fig. 1. Best example of the formation of new segments through nerve deflection on the dorsal surface of the body.

Fig. 2. Induction of new segments through implantation of a piece of ventral nerve cord.

formed by implantation. In either case the new segments thus induced are in general so poorly developed and small in number, that they can never go beyond forming something like the 'tête filiforme' of Avel (1930). That the new formation does not represent a tail end is evident both from the external appearance and from the internal structures examined upon the sections. But on the other hand, no direct evidence is easily obtained that it really represents a head end. Nevertheless, that it in all probability is a head end can only be inferred from the fact that it was induced at that level which, if cut transversely, would ordinarily regenerate a head and from the existence of a large and fairly distinct spherical ganglion comparable to a brain, which was formed by thickening of the anterior end of the nerve cord acting here as the inductive agent. It has no intestine, the interior being occupied, in spite of outer segmentation, either by mesenchymatous tissues or by a

1) Present address: Zool. Institute, Tokyo Imp. University. 
cavity, which has no septa and communicates directly with the coelome of the original segment. Induction is also possible through implantation of the brain, but a mere outgrowth with no segmentation of the dorsal wall is the result. (Formation of similar outgrowths through implantation of the brain was reported by Crowell, 1939). Hence, in earthworms, the inductive capacity of the brain is thought to be inferior to that of the ventral cord. The inductive capacity of the latter, again, is not uniform along its length. The results of our experiments indicate that it predominates at the anterior end and gradually declines toward the posterior, manifesting itself in a manner of gradients.

Now, the poorly developed head on the dorsal wall, which is obtained through induction as above described, can be made to elongate by either of the following procedures. First, one may cut off those anterior segments just before the level where the anterior end of the diverted ventral cord terminates in the wound. Then the regeneration, which has been suppressed by the presense of the head, becomes fairly enlarged. Under this condition the intestinal canal of the original worm often extends into the newer segments. When such is the case the regeneration is reinforced to a further extent and the internal structure also becomes markedly improved. The newly formed segments turn anteriorly in front of the cut surface and wholly replace the head end which was removed by the amputation. But the new and the old segments are entirely opposite as regards dorso-ventral differentiation. Here it may be remarked in passing that, in earthworms, the contact with the nerve cord is a factor which determines the dorsoventrality of the body wall.

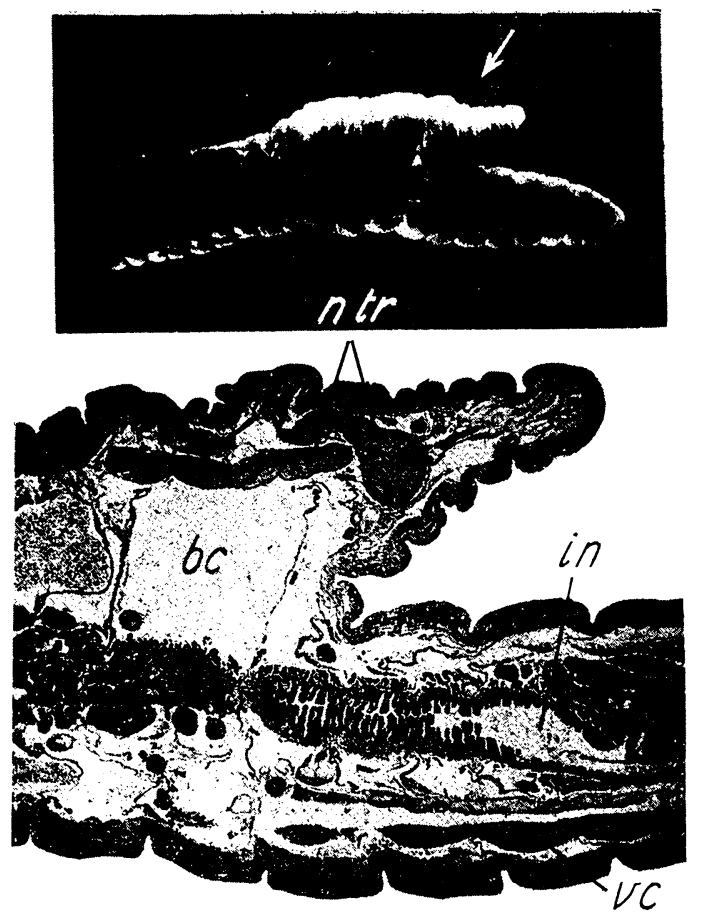

Fig. 3. Similar induction of new segments as in the preceding case through implantation of a pair of ventral nerve cords. Notice unparallel development of new segments.

Fig. 4. Median longitudial section of the same specimen as Fig. 3 through the induced segments. $b c$-body cavity, in-intestine, $\quad n$ tr-transplanted nerve cord, $v c$-ventral nerve cord. 
Another means of augmenting inductive effect is to increase the mass of the nervous substance to be implanted under the body wall. Although using of a longer piece seems to have an effect to some degree, the most effective means is in this case to increase the number of nerve cords to be implanted. We implanted two or more ventral nerve cords side by side. Then, instead of the poorly developed filiform head, there comes into appearance a head as large as or, sometimes, even larger than that of the host. In Fig. 3 is shown the result of parallel im plantation of a pair of ventral nerve cords under the dorsal wall. Comparison with Fig. 2, a single nerve cord graft, makes further explantation unnecessary as to how the induction has been reinforced. Here contrary to the case of nerve deflection and subsequent amputation the intestine has not come to extend out from the old segments. Hence, in spite of such marked development in the external appearance, as above stated, no improvement of the internal structure results: no septa are found between the segments and a common cavity as is shown in Fig. 4 communicates with the coelome of the old body. Meanwhile if, for the purpose of increasing the nervous substance, more than two ventral nerve cords are employed, no further increase in inductive effect is obtained than in the case of two. Hence it may be considered that the limit in regeneration reaction to induction on the part of the dorsal wall is reached when a pair of ventral nerve cords are used.

In the next place, if a ventral nerve cord is implanted under the dorsal wall and amputation is performed afterward at that level, cut ends of the nerve cords are exposed both at the dorsal and the ventral sides of the cut surface. Then, although the opportunity of beginning regeneration is afforded to both sides of the cut surface, the result is the same as in the ordinary case, a single head being formed (Fig. 5). This is undoubtedly due to the fact that the capacity for regeneration of the dorsal wall is far inferior to that of the ventral. Although this difference is considered by Avel (1937) to be due to some qualitative difference in the differentiation of the body wall, it must, according to our experiments, be of quantitative nature. Hence if we embed two
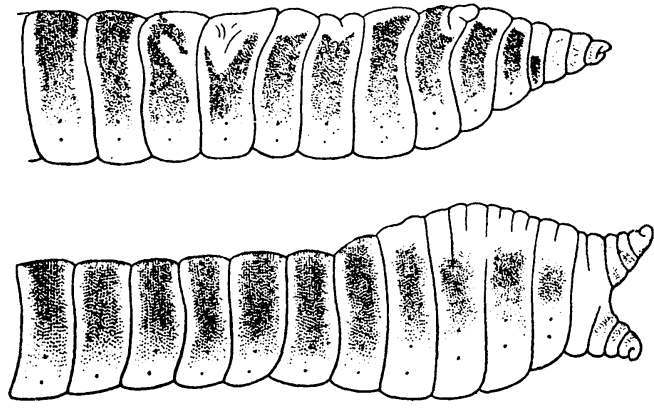

Fig. 5. Simple head regenerated from an animal operated so as to contain nerve cords both at dorsal and ventral sides of cut surface.

Fig. 6. Double head resulting from an operation similar to the preceding case but having two nerve cords at. dorsal side.

ventral nerve cords under the dorsal wall, double heads in back-to-back orientation will result. In Fig. 6 is shown a case from among such experiments. On the other hand, if a surplus ventral nerve cord is implanted along with the normal one ordinary head regeneration ensues. 
To summarize the results of above experiments on the influence of nervous substance upon the head regeneration in earthworms:

1. The ventral nerve cord is evidently a factor of inducing regeneration; its effect is only manifested at the place where its anterior cut end terminates in the wound, and not at any other intermediate portion.

2. But the situation of the nerve cord is of great significance for the dorso-ventral differentiation of the new segments and the side in direct contact with it becomes always the ventral.

3. The induction is probably of a humoral nature due to secretion of an effective substance and is evidently quantitative. Thus it can be made to correspond directly to the mass of ventral nerve cord participating, i. e., to the number of nerve cords to be implanted. Hence, by implanting two ventral nerve cords side by side the regenerative capacity of the dorsal wall, in itself small, can be made as great as or even greater than that of the ventral wall.

4. The inductive capacity of the brain is less than that of the anterior part of the ventral nerve cord. In the latter, the capacity is greatest at the anterior end and decreases toward the tail end in a manner of gradients. But the regeneration is not subject to any qualitative determination by those gradients.

5. The new segments induced on the dorsal wall constitute a mere outgrowth of the body wall, which is provided, despite external segmentation, with only a single cavity, without septa, communicating with the coelome of the original segment and containing no intestinal canal, unless a special operation is performed afterward. The brain does not develop further than a bulbar enlargement at the anterior end of the implanted nerve cord, and is not separated off from the following ganglia by connectives.

References are appended to the next paper (Part II). 